\title{
Prevalence of Left Ventricular Hypertrophy in Chronic Hemodialysis Patients of Military Hospital Mohamed V in Rabat
}

\author{
Don Carleone Sanama B ${ }^{1 *}$, Aatif Taoufiq ${ }^{1}$, Driss El Kabbaj ${ }^{1}$, Fatimazahra \\ Fikrat $^{2}$, Ilyasse Asfalou ${ }^{2}$ and Aatif Benyass ${ }^{2}$ \\ ${ }^{1}$ Department of Nephrology-Dialysis-Kidney Transplantation, Mohamed V Military \\ Training Hospital, Rabat, Morocco \\ ${ }^{2}$ Cardiology Center, Mohamed V Military Training Hospital, Rabat, Morocco \\ *Corresponding Author: Don Carleone Sanama B, Department of Nephrology- \\ Dialysis-Kidney Transplantation, Mohamed V Military Training Hospital, Rabat, \\ Morocco.
}

DOI: $10.31080 /$ ASMS.2022.06.1186

\begin{abstract}
Introduction: Cardiovascular complications are the leading cause of morbidity and mortality in hemodialysis. Left ventricular hypertrophy (LVH) is an independent factor of cardiovascular morbidity and mortality. Its frequency is 60 to $80 \%$ in chronic hemodialysis patients.

Objective: Determine the prevalence of LVH for more adequate cardiovascular management of our patients.

Methods: Observational and analytical cross-sectional study, carried out over a period of six months (February to July 2020), at the Hemodialysis Center of the HMIMV in Rabat. The study population consisted of chronic hemodialysis patients of both sexes, affiliated to the center, placed on conventional hemodialysis for more than three (3) months, and who had received pulsed and continuous Doppler echocardiography 2D. Demographic and clinical-biological data were collected from patient records, supplemented by questioning and listed on a pre-established operating sheet. LVH was defined as LV mass indexed to the body surface or LVMI (g/m2): $\geq 95$ in women and $\geq 115$ in men; The echocardiography was performed by the same operator, within 24 hours of the last dialysis session and data was collected via EchoPac software. The analysis was carried out with SPSS 20.0 software and written with Microsoft Office Word 2013.

Results: The size of our sample was 38 patients, of which $50 \%$ were men and $50 \%$ were women, for a sex ratio of 1 . The mean age was $60.21 \pm 16.864$. The mean length of time on dialysis was $106.53 \pm 72.72$. Diabetic nephropathy was the most common in $31.6 \%$ of cases. Arterial hypertension was the 2nd CVRF present in $42.1 \%$ of cases. $94.7 \%$ were on AVF dialysis, and $71.1 \%$ had distal AVF. Predialytic hypertension was present in $57.9 \%$ of patients. The mean PPID was $4.55 \pm 1.25$. The mean dry weight was $65.73 \pm 11.5$. 8 patients $(21.1 \%)$. The frequency of LVH was $31.6 \%$ with a female predominance. Concentric LVH was most common in $23.7 \%$ of cases; $36.8 \%$ had a concentric remodeling. The mean LVMI was $92.80 \pm 28.87$ with a maximum LVMI of $164.4 \mathrm{~g} / \mathrm{m} 2.31 .6 \%$ of patients had Hemoglobin $<10 \mathrm{~g} / \mathrm{dl}$ and the mean PRU was $72.94 \pm 6.45$.

Conclusion: LVH is very common in chronic hemodialysis patients. Its prevalence is $31.6 \%$ in our series. Its early diagnosis by $2 \mathrm{D}$ echocardiography must be done systematically. And its multidisciplinary management is important in order to reduce cardiovascular morbidity and mortality.
\end{abstract}

Keywords: Left Ventricular Hypertrophy (LVH); Hemodialysis; Cardiovascular Complications; HMIMV

Citation: Don Carleone Sanama B., et al. "Prevalence of Left Ventricular Hypertrophy in Chronic Hemodialysis Patients of Military Hospital Mohamed V in Rabat". Acta Scientific Medical Sciences 6.3 (2022): 20-27. 


\section{Abbreviation}

ASE: American Society of Echocardiography; AVF: Arteriovenous Fistula; BMI: Body Mass Index; BP: Blood Pressure; CHU: University Hospital Center; CKD: Chronic Renal Failure; CRP: C Reactive Protein; CVRF: Cardiovascular Risk Factors; dTDVG: Telediastolic Diameter of the Left Ventricle; EACVI: European association of Cardiovascular Imagery; ECG: Electrocardiogram; ECV: Extracellular Volume; eTDSIV: Tele Diastolic Thickness of the Interventricular Septum; eTDPP: Tele Diastolic Thickness of the Posterior Wall; F: Female; CVDF: Cardiovascular Risk Factors; LVEF: Ejection Fraction of the Left Ventricle; G: Gram; GNEC: Extra Capillary Glomerulonephritis; Hb: Hemoglobin; HD: Hemodialysis; HDL: Higth Density Lipoprotein; HMIMV: Mohamed V Military Training Hospital; HSF: Segmental and Focal Hialynosis; HTA: Arterial Hypertension; ICV: Inferior Cava Vena; L: Liter; LDL: Low Density Lipoprotein; LV: Left Ventricle; LVEF: Left Ventricular Ejection Fraction; LVH: Left Ventricular Hypertrophy; LVMI: Left Ventricular Mass Indexed; M: Male; Mg: Milligram; MHC: Hypertrophic Cardiomyopathy; MRI: Magnetic Resonance Imaging; ml: Milliliter; MHZ: Megahertz; N: Normal; $\mathrm{N}^{\circ}$ : Number; NAS: Néphroangiosclérosis; ng: Nanogram; NTIC: Chronic Tubulointerstitial Nephropathy; PAD: Diastolic Blood Pressure; PAS: Systolic Blood Pressure; PPID: Interdialytic Weight Gain; PRU: Percentage Reduction in Urea; RWT: Relative Wall Thickness; SPSS: Statistical Package for the Social Sciences; RAAS: Renin Angiotensin Aldosterone System; \%: Percentage

\section{Introduction}

Cardiovascular complications are the leading cause of morbimortality in hemodialysis; hence the interest of early screening and therapeutic management in chronic hemodialysis patients. Some of these complications appear early in the stage of chronic renal failure (CKD) before dialysis, with a prevalence that is 10 to 30 times higher than that of the general population [1]. A patient with chronic renal impairment is more likely to die from cardiovascular disease than to reach the dialysis stage [2]. Left ventricular hypertrophy (LVH) is one of the main cardiovascular complications, as well as an independent factor of cardiovascular morbidity. It is present in $16-19 \%$ of the general population, in $40 \%$ of renal failure before dialysis, and in $60-80 \%$ of chronic hemodialysis $[3,4]$. Its prevalence in Morocco is 53\% [5].
$\mathrm{LVH}$ is an increase in the volume of the left ventricle (LV) of the heart with thickening of its walls. Initially it is an adaptive response allowing the left ventricle, to maintain its capacity in the face of pressure and flow constraints to which it is subjected [6]. The increase in the mass of myofibrils is associated in the uremic, with a rarefaction of the density of the capillaries and especially with interstitial fibrosis in which participate among others: oxidative stress, hyperparathyroidism, inflammatory syndrome and uremic toxicity [7]. It leads to conduction disorders such as arrhythmias that can go as far as cardiac arrest, and diastolic dysfunction resulting from the loss of elasticity of the $\mathrm{LV}$ reducing its filling in the course of diastole.

There are two pathophysiological models of LVH according to the mechanism of parietal stress: concentric LVH and eccentric LVH (Figures 1 and 2). The LVH of chronic hemodialysis is most often mixed, this related to the association of several risk factors $[2,7]$.

Concentric LVH results from the elevation of the post-load (pressure overload), and is secondary mainly to hypertension, arteriosclerosis, calcified aortic narrowing and vascular calcifications favored by hyperparathyroidism. It is a thickening of the wall of the LV without increasing its internal diameter.

Eccentric LVH results from an increase in preload (volume overload) which is related to an increase in extracellular volume (ECV), anemia, and hyper flow of the arteriovenous fistula (AFV) especially when it is proximal. This is a dilation of the cavity of the VG. Some hydro electrolyte disorders such as hyperkalemia, metabolic acidosis; as well as activation of the renin angiotensin aldosterone system (RAAS), also participate significantly in the development of LVH and myocardial fibrosis [3].

There are also classic non-hemodynamic factors such as: age, gender, race that could be incriminated. A study done in 2008 in Colorado in the USA, highlighted a predominance of hypertension-related LVH in the African-American population compared to the Caucasian population, i.e. $69 \%$ versus $66 \%$ [8]. 
MRI remains the best tool for detecting and quantifying LV abnormalities, but Echocardiography also has a prominent place in the diagnostic and therapeutic management of $\mathrm{LVH}$ and requires the measurement of left ventricular mass indexed (LVMI) [9] (Figure 1). This measurement should take into account the rapid variations in volume in chronic hemodialysis [2]. The place of echocardiography is essential because it makes it possible to distinguish it from hypertrophic cardiomyopathy (MHC) and other etiologies such as cardiac amyloidosis and the heart of the athlete. In the latter case, the ECG alone is inconclusive to retain the diagnosis of LVH [4,10-12].

\section{Objective}

It was a question of determining the prevalence of LVH, for a more adequate management of our patients on the cardiovascular level.

\begin{tabular}{lcc}
\hline Linear method & & \\
LV mass $(\mathrm{g})$ & $67-162$ & $88-224$ \\
LV mass/BSA $\left(\mathrm{g} / \mathrm{m}^{2}\right)$ & $43-95$ & $49-115$ \\
Relative wall thickness $(\mathrm{cm})$ & $0.22-0.42$ & $0.24-0.42$ \\
Septal thickness $(\mathrm{cm})$ & $0.6-0.9$ & $0.6-1.0$ \\
Posterior wall thickness $(\mathrm{cm})$ & $0.6-0.9$ & $0.6-1.0$ \\
2D method & & \\
LV mass $(\mathrm{g})$ & $66-150$ & $96-200$ \\
LV mass $/$ BSA $\left(\mathrm{g} / \mathrm{m}^{2}\right)$ & $44-88$ & $50-102$ \\
\hline
\end{tabular}

Figure 1: Normal range of measurement of LV mass indexed [4].

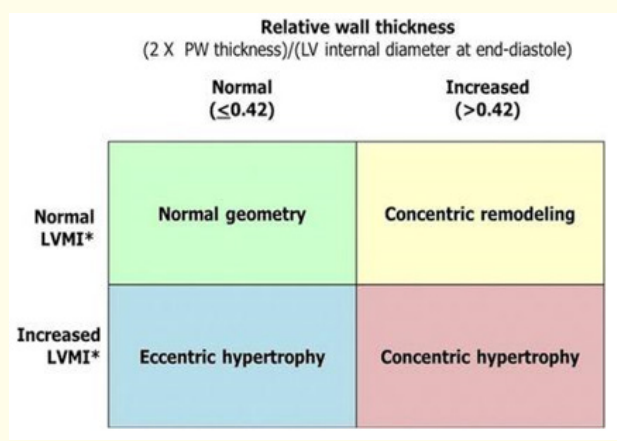

Figure 2: Geometry of LV [4].

\section{Methods}

This is an observational and analytical cross-sectional study, which took place over a period of six (06) months (February to July 2020), at the Hemodialysis Center of the Mohamed V Military Training Hospital in Rabat. Our study population consisted of chronic hemodialysis patients treated at this center.

All patients of both sexes who have been on conventional hemodialysis (HD) for more than three (3) months, and who have received 2D transthoracic ultrasound with pulsed and continuous Doppler have been included. Patients recently placed on hemodialysis ( $<3$ months) and those who had emergency dialysis were not included. Demographic and clinical-biological data were collected from patient records, supplemented as necessary by patient interrogation, and listed on a pre-established operating record.

These were: age, gender, seniority on dialysis; initial nephropathy; the type of vascular first and its flow; comorbidities and CVRF; functional signs; biological parameters (hydro-electrolytic; mineralo-bone; nutritional, inflammatory, lipid, hematological; nitrogen retention and dialysis quality). The echocardiographic parameters studied were: cardiac structures (walls, diameter, systolic function and global and segmental contractility of the LV and RV; surface of the atria); valve structures (kinetic, calcifications), flows (mitral, aortic, pulmonary, tricuspid, filling pressure of LV), pericardium and inferior vena cava (IVC).

LVH was defined by the mass of LV indexed to body surface or LVMI $\left(\mathrm{g} / \mathrm{m}^{2}\right)$ : $\geq 95$ in women and $\geq 115$ in men. It was calculated according to the latest joint recommendations of the American Echocardiography Society (ASE) and the European Cardiovascular Imaging Association (EACVI) according to this formula (linear method): Mass of LV $=0.8 \times\{1.04 \times[(d T D V G+$ eTDSIV + eTDPP $) 3$ - (dTDVG)3] $\}$ + $0.6 \mathrm{~g}$ (dTDVG: telediastolic diameter of the left ventricle; eTDSIV: telediastolic thickness of the interventricular septum; eTDPP: telediastolic thickness of the posterior wall). It was calculated with the patient's theoretical dry weight. When the mass was increased, the geometry was characterized as concentric or eccentric by calculating the relative thickness of the walls (Figure 2); according to the formula: RWT $=2$ XeTDPP/dTDVG. If RWT $>0.42$ : 
it was a concentric geometry (increase in wall thickness/cavity size ratio); if RWT $\leq 0.42$ : it was an eccentric geometry. In case of normal mass with a relative thickness $>0,42$, it was called concentric remodeling [4].

The transthoracic ultrasound was performed with a VIVID 9 Vingmed system V (General Electric Medical System) ultrasound system and an M5S-D probe with a frequency oscillating between 1 and $8 \mathrm{MHZ}$. It was done by the same operator, and within 24 hours of the last dialysis session to avoid biases related to variations in the ECV. Echocardiographic data were collected via EchoPac software.

Blood pressure (BP) was taken at rest in a sitting position, then in the supine position at the beginning of the session and on the contralateral side at the vascular approach. Arterial hypertension was defined by a blood pression $\geq 140 / 90 \mathrm{mmHg}$. An hemoglobin threshold $<10 \mathrm{~g} / \mathrm{dl}$ was considered pathological and a CRP $>5.3$ $\mathrm{mg} / \mathrm{l}$ was said to be positive. Cardiomegaly was defined by a cardiothoracic index $\geq 0.55$ (ratio of the greatest width of the thoracic silhouette to the greatest width of the cardiac silhouette). The LVEF was retained if $>45 \%$. Hyper phosphoremia and hypocalcemia were retained in front of these respective levels: $>50 \mathrm{mg} / \mathrm{l}$ and $<80 \mathrm{mg} / \mathrm{l}$.

The data analysis was done from the SPSS 20.0 software and the input with Microsoft Office Word 2013. Quantitative variables were expressed as averages \pm standard deviation and qualitative variables in numbers and percentages.

\section{Results}

\section{General population data}

Our sample size was 38 chronic hemodialysis patients.

\section{Sociodemographics data}

The age of the patients ranged from 24 to 86 years, with an average age of $60.21 \pm 16.864$. The predominant age range was 61 to 80 years. The sample consisted of $50 \%$ men and $50 \%$ women, a sex ratio of 1 .

\section{Dialytics data}

All patients were on conventional hemodialysis. Seniority on dialysis ranged from 13 to 280 months. 23 patients $(60.5 \%)$ were under 10 years of age on dialysis. The dominant initial nephropathy was diabetic nephropathy found in 12 patients (31.6\%). Concerning the vascular approaches: $94.7 \%$ were dialysis by AVF, and $5.3 \%$ by tunelized jugular catheter; $71.1 \%$ had a distal AVF.

\section{Comorbidities and CVRF}

29 patients $(76.3 \%)$ had at least one comorbidity. Diabetes and heart disease were the most common, respectively: 31.6\% (12 cases) and $28.9 \%$ (11 cases); followed by PAD and neoplastic pathology or $10.5 \%$ for each. $76.3 \%$ (29 patients) had at least one CVRF. Sedentary living and hypertension were the most common CVRF, respectively: $60.5 \%$ and $42.1 \%$.

\section{Clinicals and para-clinicals data}

Clinics

$42.1 \%$ of patients were symptomatic (dyspnea, palpitations, angina). Predialytic hypertension was present in $57.9 \%$ of patients. PPID (inter dialytic weight gain) ranged from 1 to $4 \mathrm{~kg}$. 8 patients (21.1\%) had PPID>3 kg. 42.9\% had normal BMI and $14.3 \%$ had morbid obesity.

\section{Paraclinics}

$68.4 \%$ of patients had a residual diuresis ranging from 100 to $2000 \mathrm{ml}$.

About the phosphocalcium assessment: Hypocalcemia was found in 10 patients (26.3\%), and 52.6\% had significant hyperparathyroidism $(\geq 10 \mathrm{XN}) .31 .6 \%$ of patients had $\mathrm{Hb}<10 \mathrm{~g} / \mathrm{dL}$ and $47.4 \%$ had elevated CRP. Dyslipidemia was observed in $18.4 \%$ of cases (7 patients), and $21.9 \%$ of patients were undernourished.

Regarding echocardiographic data: Cardiomegaly was present in $31.6 \%$ of patients. The LVMI maximum was $164.4 \mathrm{~g} / \mathrm{m}^{2}$. LVEF was retained in $97.4 \%$ of cases, and ICV was fine and compliant in $97.4 \%$ of cases.

\section{Frequency of LVH in chronic hemodialysis patients}

The frequency of LVH in chronic hemodialysis patients at the HMIMV in Rabat was $31.6 \%$ (12 patients), or 7 women (58.3\%) versus 5 men (41.7\%). Concentric LVH was predominant (23.7\%) compared to eccentric (7.9\%). Concentric remodeling was described in 14 patients or $36.8 \%$. 


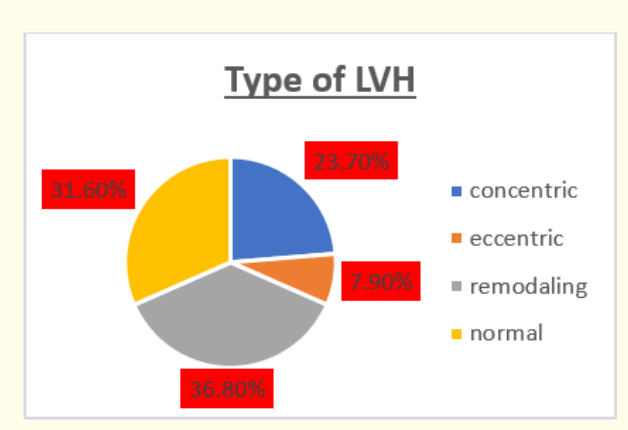

Figure 3: Distribution of the population by type of LVH.

\begin{tabular}{|c|c|}
\hline Middle age (year) & $60,2 \pm 16,86$ \\
\hline Sex ratio $(M / F)$ & 1 \\
\hline Duration in HD (month) & $136,32 \pm 22,93$ \\
\hline Dry weight (Kg) & $65,73 \pm 11,49$ \\
\hline PPID (Kg) & $4,55 \pm 1,25$ \\
\hline $\mathrm{Kt} / \mathrm{V}$ & $1,54 \pm 0,23$ \\
\hline PRU (\%) & $72,94 \pm 6,45$ \\
\hline Beta2microglobulin (mg/l) & $44,56 \pm 18,33$ \\
\hline PAS (mmHg) & $136,32 \pm 22,93$ \\
\hline PAD (mmHg) & $73,58 \pm 19,5$ \\
\hline \multicolumn{2}{|l|}{ Initial Néphropathy (\%) } \\
\hline Diabetes & 31,6 \\
\hline NTIC & 26,3 \\
\hline Glomerular Nephropathy & 7,9 \\
\hline Indeterminate & 26,3 \\
\hline Others* (NAS, HSF, GNEC) & 7,8 \\
\hline Proximal AVF (\%) & 23,6 \\
\hline Distal AVF (\%) & 71,1 \\
\hline \multicolumn{2}{|l|}{ CVRF (\%) } \\
\hline Obesity & 15,8 \\
\hline Sedentary lifestyle & 60,5 \\
\hline Dyslipidemia & 18,4 \\
\hline HTA & 42,1 \\
\hline Smoking & 5,3 \\
\hline
\end{tabular}

Table 1: Socio-demographics and dialytics data.

\begin{tabular}{|c|c|}
\hline Parameters & Moyenne \pm écart-type \\
\hline LMVI $\left(\mathrm{g} / \mathrm{m}^{2}\right)$ & $92,8 \pm 28,87$ \\
\hline LVEF $(\%)$ & $59,74 \pm 6,66$ \\
\hline Kalemia & $5,24 \pm 0,73$ \\
Alkalines reserves (mmol/l) & $21,42 \pm 2,7$ \\
Urea (g/l) & $1,11 \pm 0,265$ \\
Creatinine (mg/l) & $91,84 \pm 23,12$ \\
\hline Pthi (pg/ml) & $732,4 \pm 532,47$ \\
Serum Calcium (mg/l) & $88,5 \pm 9,51$ \\
Phosphorémia (mg/l) & $44,47 \pm 15,31$ \\
CRP (mg/l) & $12,28 \pm 15,49$ \\
Albumin (g/l) & $38,53 \pm 4,56$ \\
Hémoglobin (g/dl) & $10,9 \pm 1,41$ \\
HDL cholestérol (g/l) & $0,39 \pm 0,98$ \\
Ferritinémia (ng/ml) & $423,08 \pm 270,99$ \\
25 (OH) Vitamin D (ng/ml) & $32,45 \pm 20,64$ \\
\hline
\end{tabular}

Table 2: Biologicals and Echocardiographics parameters.

\section{Discussion}

\section{Frequency of LVH}

LVH represents one of the common cardiovascular complications, as well as an independent factor of cardiovascular morbidity in patients with renal impairment before dialysis (40\%), and in chronic hemodialysis in particular (60-80\%) [3,4]. Several studies have been done on this subject in Africa, particularly in Morocco, Benin, Tanzania and other continents.

In our series, the frequency of LVH was $31.6 \%$ with a female predominance (18.43\%). 2 studies done in Morocco on the one hand in 2013 at the University Hospital of Oujda on 70 patients, and on the other hand in 2014 at the Hassan II University Hospital of Fez on 50 patients found a prevalence of LVH respectively of $53 \%$ and $56 \%[5,13]$. This is also the case in the Vigan series in Benin which found LVH in 77 out of 141 chronic hemodialysis patients, or $54.6 \%$ [6].

In a Tanzanian cohort study done in 2017, this prevalence was $42 \%[14]$. 
This discrepancy in the frequency of LVH compared to other studies could be explained by the small size of our sample.

In general, the risk of cardiovascular disease is low in women. A New York study conducted in 2017, which aimed to test whether this sex-related protection played a role in the occurrence of LVH even in the presence of hypertension, found a predominance of $\mathrm{LVH}$ in women (43.4\% versus $32.1 \%$ for men) [15]. This is consistent with our results.

Concentric LVH was predominant in our series at $23.7 \%$. It should also be noted that the significant rate of patients with concentric remodeling was $36.8 \%$. These results are similar to those of Vigan which also found in its series a concentric HVG in $54.6 \%$ and a concentric remodeling in 45.4\% [6]. A 1996 Canadian cohort of 432 patients recently placed on hemodialysis also found the predominant concentric LHV at 41\% [16]. This could be explained in our study by the considerable prevalence of hypertension (42.1\%), which is implicated mainly in the pathophysiology of this type of LVH resulting from the elevation of the post-load.

\section{Dialytics data and CVRF}

The average length of time on dialysis was $106.53 \pm 72.72$. These data support those found by Eziani., et al. who reported an average of $93 \pm 55$ [13]. 23 patients or $60.5 \%$ had less than 10 years on dialysis, compared to $20.6 \%$ in the Vigan series in Benin [6].

The dominant initial nephropathy was diabetic nephropathy found in 12 patients (31.6\%). On the other hand, in Kharimi., et al. in Oujda, diabetic nephropathy accounted for 13\% [5]. In the series of Eziani., et al. in Fez and Faqih SA at the CHU Ibn Roch in Casablanca, nephropathy was indeterminate in the majority: respectively $42 \%$ and $46 \%[13,17]$.

AVF was the most common vascular onset in $94.7 \%$ of cases or 36 patients and $71.1 \%$ had distal AVF. Eziani., et al. $80 \%$ of hemodialysis patients had distal AVF [13]; which is consistent with the results of our study.

Sedentary living and hypertension were the most common CVRF, respectively: $60.5 \%$ and $42.1 \%$. These data are similar to those found in the Tanzanian cohort that highlighted the presence of hypertension in $60 \%$ of patients [14]. Hypertension itself is a common cardiovascular disease and is a major modifiable risk factor for cardiovascular disease [18].

\section{Clinicals and paraclinicals data}

Pre dialytic hypertension was present in $57.9 \%$ of patients. In a Beninese study done at the CNHU-HKM in Cotonou, pre dialytic blood pressure was elevated in $46.8 \%$ of cases [6]. This is statistically close to our data, and could be explained by the high frequency of hypertension constituting the second CVRF in our series, at a rate of $42.1 \%$.

PPID ranged from 1 to $4 \mathrm{~kg}$ with an average of $4.55 \pm 14.25$. 8 patients (21.1\%) were overloaded (PPID>3 kg), however in Fez, Eziani found an average of $2.2 \pm 0.7$ [13]. Numerous studies have shown that PPID correlates with interdialytic interval, the longer it is, the more PPID increases and is associated with a high risk of cardiovascular morbidity. A New York study done in 2016, demonstrated that intensive daily hemodialysis through short night sessions significantly reduced the mass of LV compared to conventional (3 weekly sessions) [19].

Regarding the dialysis dose, the average PRU $=72.94 \pm 6.45$; which correlates with the result found by Vigan: $72.8 \pm 11.8 \%$ [6].

Anemia was present in $31.6 \%$ of cases with an average of 10.90 \pm 1.41 . Faqih SA in casablanca and Kharimi., et al. in Oujda found anemia in $41 \%$ and $78 \%$ respectively $[5,17]$. This low level compared to other studies could be explained by the rigorous management of our patients, who was systematically put on erythropoiesis stimulating agent as soon as the hemoglobin level is $<10 \mathrm{~g} / \mathrm{dl}$; with blood count monthly control for therapeutic adjustment.

Cardiomegaly was present in $31.6 \%$ of patients, on the other hand in Vigan in Benin, it was observed in 55.4\% [6].

\section{Conclusion}

Left ventricular hypertrophy (LVH) is a very common pathology in hemodialysis and is a major predictor of cardiovascular morbidity. Its prevalence is $31.6 \%$ in our series, with a female predominance or $18.43 \%$. Its early diagnosis by 2D echocardiography 
during the annual cardiovascular assessment, must be done systematically. Its multidisciplinary management involving cardiologists and nephrologists is necessary to reduce the risk of cardiovascular morbidity particularly high in this population.

\section{Conflicts of Interest}

The authors do not declare any conflict of interest.

\section{Authors' Contributions}

All of the above-mentioned authors participated in the development of this work. They all read and approved the final and handwritten version.

\section{Bibliography}

1. Hassani K., et al. "Echocardiographic abnormalities in chronic end-stage renal failure Causes of death of dialysis patients: descriptive epidemiology 2001 / 2010 Bioethics Law 2004 and donation of life: the word to donors Urinary and hypert abnormalities". 7.2011 (2020): 2010-2011.

2. Vircoulon M and Combe C. "Cardiac consequences of chronic renal failure”. 18.12 (2020): 1-14.

3. Taufiq Rohman S.Pd.I MP. "le Manuel du Résident". Psikol Perkemb (2019): 1-224.

4. Suisse D and Finalement H. "Discovery of left ventricular hypertrophy on adult echocardiography". Revue Médicale Suisse 13 (2017): 1106-1112.

5. Karimi I., et al. "Prevalence and predictive factors of occurrence of left ventricular hypertrophy in chronic hemodialysis Anxiodepressive disorders in chronic hemodialysis in eastern Morocco Ophthalmological manifestationss". Nephrology Therapy 9.5 (2013): 290.

6. Vigan J., et al. "Left ventricular hypertrophy in chronic hemodialysis patients at CNHU-HKM of Cotonou". Nephrology Therapy 14.1 (2018): 29-34.

7. Gosse P., et al. "Hypertrophie ventriculaire gauche au cours de l ' hypertension artérielle". EMC - Cardiology 10.15 (2015): $1-11$.
8. Havranek EP., et al. "Left Ventricular Hypertrophy and Cardiovascular Mortality by Race and Ethnicity". The American Journal of Medicine 121.10 (2008): 870-875.

9. Glassock RJ., et al. "Left Ventricular Mass in Chronic Kidney Disease and ESRD". 7 (2009): 79-91.

10. Chez É and Hd LH. "L'échocardiographie chez l'hémodialysé chronique".

11. Malík J., et al. "Echocardiography in patients with chronic kidney diseases”. Cor Vasa 60.3 (2018): e287-295.

12. Hedman K., et al. "Limitations of Electrocardiography for Detecting Left Ventricular Hypertrophy or Concentric Remodeling in Athletes". The American Journal of Medicine 133.1 (2020): 123-132.e8.

13. Ezziani M., et al. "Echocardiographic abnormalities in chronic hemodialysis: Prevalence and risk factors". Pan African Medical Journal 18 (2014): 1-6.

14. Raphael DM., et al. "Heart diseases and echocardiography in rural Tanzania: Occurrence, characteristics, and etiologies of underappreciated cardiac pathologies". PLoS One 13.12 (2018): 1-16.

15. Gerdts E., et al. "Left ventricular hypertrophy offsets the sex difference in cardiovascular risk (the Campania Salute Network)". International Journal of Cardiology 258 (2018): 257261.

16. Parfrey PS., et al. "Nephrology Dialysis Transplantation Outcome and risk factors for left ventricular disorders in chronic uraemia" (1996).

17. Faqih SA., et al. "Evaluation of Phosphocalcium, Nutritional, Cardiovascular and Complications Of Vascular Approach In Hemodialysis Over 20 Years". Nephrology and Therapeutics 11.5 (2015): 306.

18. Yildiz M., et al. "Left ventricular hypertrophy and hypertension". Progress in Cardiovascular Diseases 63.1 (2020): 10-21. 
19. McCullough PA., et al. "Intensive Hemodialysis, Left Ventricular Hypertrophy, and Cardiovascular Disease". American Journal of Kidney Diseases 68.5 (2016): S5-14.

\section{Assets from publication with us}

- Prompt Acknowledgement after receiving the article

- Thorough Double blinded peer review

- Rapid Publication

- Issue of Publication Certificate

- High visibility of your Published work

Website: www.actascientific.com/

Submit Article: www.actascientific.com/submission.php

Email us: editor@actascientific.com

Contact us: +919182824667 